Comme l'on met de ro à r 5 pour 100 d'eau pour constituer le mortier, cela revient, avec une addition de 2 pour 100 de chlorure de calcium, à gâcher avec une eau contenant de 13 à 20 pour 100 de chlorure, ce qui abaisse le point de congélation de cette eau à $-10^{\circ} \mathrm{C}$ et $-18^{\circ} \mathrm{C}$.

Il est à remarquer, d'une part, qu'une adjonction de chlorure supérieur à 2 pour ıoo augmente la rapidité de la prise et que, d'autre part, les ciments sont d'autant moins résistants que la rapidité de la prise est plus grande.

Pour vérifier l'augmentation de l'imperméabilité du mortier par l'adjonction de chlorure de calcium, M. Meade fit sécher dans l'air de petites plaques de mortier à $\mathrm{I}: 3$ pendant deux semaines, puis les plaça dans un vase contenant de l'eau, et les y laissa 24 heures. Des pesées, effectuées avant et après cette immersion, accusaient la quantité d'eau absorbée. M. Meade trouva que le mortier sans addition de chlorure absorbait 7,5 pour 100 d'eau, tandis que le mortier à 2 pour I oo de chlorure n'en absorbait que 3,7 pour roo, soit une diminution de moitié.

Des plaques de mortier, contenant 4 et 6 pour roo de chlorure de calcium, présentèrent des efflorescences, ce qui n'eut pas lieu avec une proportion de 2 pour soo seulement. Il est à remarquer que, à cause de l'affinité du chlorure de calcium par l'eau, le mortier additionné de ce sel est plus long à sécher qu'un mortier pur.

\section{CONGRĚS DE BORDEAUX DES TRAVAUX PUBLICS}

Le Congrès national des Travaux publics s'est réuni cette année à Bordeaux, dans le courant de juillet, sous la présidence de M. PREvwr, sénateur. Le Congrès a adopté une série de vœux relatifs à l'amèlioration du régime des chemins de fer et de la navigation en France. Au cours de ce Congrès M. Millerand a prononcé le discours suivant :

\section{Messieurs,}

J'ai eu l'honneur imprévu et le grand avantage de présider aux travaux de vos première et cinquième sections réunies, chargées d'étudier l'une l'amélioration des ports de commerce, l'autre la personnalité civile des grandes cuvres d'utilité publique.

A ce titre, je vous demande la permission de vous soumettre en manière de préface à la discussion et au vote des vœux qui vous sont présentés quelques observations très générales.

En lisant les documents si intéressants rassemblés en vue de ce congrès, vous avez été à coup sûr comme moi frappés des constatations affligeantes qui y réapparaissent comme un refrain.

Un demi-siècle ne s'est pas écoulé et notre port de Marseille - pour ne citer que celui-là - qui occupait sur la liste des grands ports d'Europe le second rang est tombé au sixième. Certes le mouvement des entrées s'y est sans cesse accru, mais sur un rythme dépassé de loin par celui des cités rivales : et Anvers passant de 546.000 tonneaux en I 860 à 9.816 .000 en 1905 , et Hambourg bondissant, dans la même période, de 948.000 tonneaux à 10.400 .000 .

De même le mouvement total de nos échanges n'a cessé de croître, mais combien celui de nos rivaux a augmenté plus rapidement! En trente ans, l'Allemagne et les Etats-Unis nous ont rejoints et largement distancés, et nous sommes serrés de près par les Pays-Bas.

Quant à la décadence de notre marine marchande, des débats réitérés et récents ne nous laissent aucune illusion sur sa triste réalité.

Devant de telles constatations, que faire ? Gémir et se rési- gner? Non, certes. Regarder en face le mal, trouver le remède et l'appliquer.

A quelles causes attribuer l'infériorité de nos ports, l'atonie de notre système circulatoire? Comment un pays qui semble désigné par la nature comme le passage nécessaire entre le centre de l'Europe et les pays d'outre-mer, comment le pays de la Loire et du Rhône en est-il arrivé à voir se créer en dehors de lui, donc contre lui, des courants commerciaux si malaisés ensuite à rectifier; pourquoi nos commerçants et nos industriels en sont-ils encore à réclamer des travaux publics dont la nécessité saute aux yeux? On l'a dit et l'on vous le redira.

Je ne veux pour ma part faire le procès ni de notre régime électoral - c'est ici un domaine interdit - ni de notre addministration : il est trop aisé d'en médire, plus sans doute que d'en user, car c'est un merveilleux outil de travail pour qui veut s'en servir.

$\mathrm{Ne}$ nous attardons pas à des critiques stériles. Allons droit au remède.

C'est, en sept ans, la troisième fois que se réunit le congrès national des travaux publics français. C'est la troisième fois qu'il le recommande. Il se nomme d'un nom qui en dit trop long et pas assez : l'autonomie des ports.

Trop, car il ne s'agit pas de faire de nos ports des Etats dans l'Etat, isolés du reste du pays et indifférents à l'ensemble des intérêts nationaux. Pas assez, car ce n'est pas en vue des seuls ports, mais aussi des voies navigables qui sont leurs affluents nourriciers et d'une manière générale des grands travaux publics que ce procédé doit être recommandé.

En quoi consiste-t-il? A donner à I'initiative des intéressés directs : chambres de commerce, municipalités, conseils généraux, syndicats autonomes où seront représentés les intérêts locaux, régionaux, nationaux, le droit de s'affirmer avec les corollaires nécessaires à toute initiative qui veut aboutir : la responsabilité, les charges, mais aussi l'administration et le contrôle.

L'idée n'est pas neuve : elle a fait son chemin déjà dans les milieux commenciaux, industriels, politiques. Il faut la faire pénétrer dans le grand public. Il faut lui conquérir la puissance sans laquelle il n'est point de succès durable sous un régime de suffrage universel : l'opinion publique.

C'est votre tâche et ce sera votre honneur. Montrez à ce pays l'exemple de l'Angleterre, de la Belgique avec Anvers, de la Hollande avec Rotterdam, de l'Allemagne avec Brême et Hambourg, de l'Italie enfin avec Gênes dont le consortium nous offre un modèle si propre à être imité et si digne d'être suivi.

Ils savent bien, nos rivaux, que le bassin ou le quai construit dans un port ne doit point viser à la perfection d'un objet d'art dont on peut dire que le temps ne fait rien à l'affaire. Il n'atteint son but au contraire, il ne remplit sa fonction que si, construit vite et au meilleur marché possible pour la. satisfaction des besoins d'aujourd'hui, il peut être remplacé aussi aisément et aussi vite pour la satisfaction des besoins de demain.

Bon pour nous d'avoir mis quatorze ans à nous décider à entreprendre au Havre des travaux devenus insuffisants à l'heure même où l'on se décidait enfin à les commencer.

Il faut rompre, et sans délai, avec ces routines meurtrières. Que nous puissions faire aussi bien et mieux que nos rivaux, nous en avons, sur tous les points du globe, la preuve écrite, dains tant de ports étrangers, de la main de nos entrepreneurs et de nos ingénieurs.

Une nation aussi riche que nous le sommes de ressources et d'hommes n'a pas le droit d'être pessimiste. L'avenir nous appartient, si nous voulons seulement passer de la parole à l'acte, et réaliser nos conceptions.

Il ne s'agit certes pas pour l'Etat de se désintéresser des grandes entreprises, mais au contraire de les encourager, en stimulant, en aidant l'initiative des intéressés.

Ce qui s'agite en ce débat, sous ces problèmes en apparence exclusivement téchniques, c'est l'avenir mểme de notre Fran'- 
c'est sa grandeur morale intimement liée à sa prospérité économique. Pour remplir toute sa destinée, pour continuer d'être la grande initiatrice de la justice sociale comme de la liberté politique, notre démocratie a besoin d'être forte à tous les points de vue.

Puissance économique, puissance militaire : l'une et l'autre sont également nécesaires à un peuple qui ne veut pas déchoir.

Rien certes n'est perdu, mais il est temps et grand temps de nous ressaisir.

Parlez, agissez surtout et, j'en ai la confiance, vous serez entendus et suivis.

\section{LE MOIS HYDRO-ÉLECTRIQUE}

\section{ACADÉMIE DES SCIENCES}

\section{CHIMIE ET ÉLECTROCHIMIE}

Sur l'oxydation électrolytique du platine. - Nore de M. C. Marre, séance du 8 juillet 1907.

Un certain nombre de propriétés du platine, notamment son attaque sous l'influence du courant alternatif (de la Rive, Ruer, etc.) et son action catalytique (Engler et $\mathrm{L}$. Wohler, Traude, etc.), ont conduit à supposer que, dans les conditions expérimentales indiquées, le métal était susceptible de s'oxyder au moins superficiellement. De plus, Kohlrausch, dans une étude sur l'électrolyse des solu ions de chlorure de platine (Wied. Ann., t. LXIII, p. 423), avait remarqué l'apparition sur les anodes d'une teinte brune, totalement ćliminee au rouge, et qu'il attribuait à un superoxyde par analogie avec ceux que donnent le plomb et l'argent. Kohlrausch n'avait cependant pu constater, dans ces conditions, que les anodes variaient de pjids.

Ayant eu également l'accasion fréquente de remarquer cette même coloration desanodes le platine, j'ai pu mettre en évidence, d'une manière complète, cette oxydation superficielle.

J'ai constaté, en effet, que les anodes ainsi brunies, traitées par une solution même très titendue d'iodure de potassium, acjdulée par l'acide chlorhydrique, coluraient cette solution en un rouge plus ou moins intense, absolument comparable à la teinte que l'on obtient quand on traite une solution étendue de chlorure de platine par l'iodure de potassium. De plus, dans cette réaction des anodes, une certaine quantité d'iode semble mise en liberté, et peut se mettre en évilence au moyen du chloroforme.

J'ai pu, dans les solutions colorées ainsi obtenues, caractériser le platine, et même le doser colorimétriquement par comparaison avec des solutions connues de chlorure traitées exactement de la même manière (1). Les résultats ont été complètement d'accord arec ceux fournis nar la pesée des électrodes.

Ces.variations de poids sont d'ailleurs faibles, ainsi que le montrent les risultats suivants obtenus sur quatre électrodes ayant I00 $\mathrm{cm}^{2} ; 40 \mathrm{~cm}^{2}, 6 \mathrm{~cm}^{2}$, et $0,3 \mathrm{~cm}^{2}$, qui avaient servi danodes pendant 12 heures, avec un courant de 2 ampères dans une solution d'acide sulfurique normal; les pertes de poids respectives observées après le traitement à $\mathrm{KI}+\mathrm{HCI}$ ont été de $\frac{8}{10}, \frac{5}{10}, \frac{2,5}{10}$ et $\frac{\mathrm{J}}{10} \mathrm{de}$ milligramme.

Quant aux augmentations de poids primitives dues à la fixation de l'oxygène, elles ont toujours été constatées nettement sur les anodes d'une surface assez grandes pour que leur perte de poids ultérieure atteigne $0,5 \mathrm{mg}$. Les cathodes pesées comparativement n'ont jamais changé de poids.

Les mêmes résultats ont été obtenus par l'électrolyse de solutions des acides azotique, phosphorique, chlorhydrique ou des solutions alcalines $(\mathrm{NaOH})$; les concentrations employées étaient du méme ordre que celle de l'acide sulfurique précédemment utilisé.

La pellicule d'oxyde formée est insoluble dans $\mathrm{Az} \mathrm{O}^{3} \mathrm{H}$ : elle disparaît totalement au rouge et ne se dissout dans l'acide chlorhydrique étendu qu'en présence d'iodure. La mise en liberté d'iode semble indiquer que cet oxyde est un peroxyde; il convient cependant d'ajouter que cette dernière réaction n'est parfaitement nette qu'avec une anode recouverte de.noir de platine dont l'affinité toute particulière pour l'oxygène a été mise en évidence par C. Fugler et L. Wohler (Z.f. anorg. Ch.t. XXIX, Ig02, p. 5).

Quelle que soit, d'alleurs, la nature réelle de cet oxyde, les faits obtenus montrent nettement que, contrairement à l'opinion généra-

(1) Cette réaction du platine est d'une sensibilité considérable; la coloration rose se manifeste nettement encore pour une solution qui ne contíent. par litre, pas plus de $\theta, 0003$ de métal.

lement admise, le platine, mème en lame, est susceptible de s'oxyder facilement sous l'influence du courant continu.

Des expériences en cours d'exécution me font penser que cette oxydabilité est encore plus considérable et que, même en l'absence de courant, le platine s'oxyde superficiellement par simple contact à froid avec des solutions de corps oxydants comme le permanganate ou le persulfate de soude; les expériences, plus délicates encore que les précédentes, nécessitées par ces recherches, seront publiées ultérieurement. Quant aux documents bibliographiques concernant cette question, et aux expériences détaillées qui justifient les conclusions précédentes, ils seront donnés dans un autre recueil.

Sur les propriétés et la constitution des aciers au tantale. Note de M. L. Guillet, séance du 29 juillet 1907 .

On a beaucoup parlé des aciers au tantale, en en vantant les qualités; différents brevets, pris notamment en Allemagne et en France, donnent à entendre que l'addition de tantale améliore considérablement les propriétés des aciers.

Nous avons pensé qu'il était intéressant de faire quelques essais en vue de préciser ces qualités. A cet effet, les acieries d'Emphy ont préparé, sur notre demande, les quatre aciers suivants:

\begin{tabular}{|c|c|c|c|c|c|}
\hline $\begin{array}{c}\text { Carbone } \\
0,120\end{array}$ & $\begin{array}{c}\text { Tantale } \\
0,00\end{array}$ & $\begin{array}{c}\text { Manganèse } \\
\text { o, I }\end{array}$ & $\begin{array}{l}\text { Silicium } \\
\quad 0, I_{2}\end{array}$ & $\begin{array}{l}\text { Soufre } \\
\text { traces }\end{array}$ & $\begin{array}{c}\text { Phosphove } \\
\text { traces }\end{array}$ \\
\hline 0,170 & 0,15 & 0,15 & 0,19 & 》) & 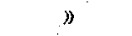 \\
\hline 0,180 & 0,60 & 0,22 & 0,24 & ע & ) \\
\hline 0,160 & 1,05 & 0,23 & 0,16 & i) & ) \\
\hline
\end{tabular}

On remarquera que les teneurs en carbone, manganèse et silicium. sont très voisines, et que la iseule variation importante dans la composition a trait au pourzentage de tantale qui varie de 0,09 à $1,0.5$ pour 100.

Ces aciers n'ont donné lieu à aucune difficulté particulière dans leur fusion, ni dans leur traitement mécanique.

La matière premièiè utitisée était un fêrro-tantale "préparé au four électrique.

A. Aclers normaux. - Micrographie. Les aciers normaux sont tous perlitiques, et ne présentent aucune particularité; cependant, la ferrite de ces aciers laisse voir quelques taches, légèrement brunes, sous l'action de l'acide picrique. La quantité de perlite est bien en en rapport avec la quantité de carbone, mais elle est d'autant mieux répartie que la teneur en tantale est plus forte.

Propriétés mécaniques.:- Le tableau suivant résume les propriétés mécaniques des aciers au tantale examinés :

Composition

$\mathrm{C}$ Ta

0,120

0,170

$0,160 \quad$ I, 05

Ces résultats montrent que le tantale prodüit une légère augmentation de la charge de rupture et de la limite d'élasticité, une faible diminution dans les allongements.

La résistance au choc est élevée.

Il est incontestable que l'effet obtenu avec une addition de I pour Ioo de tantale peut être produit avec un grand nombre d'autres corps, notamment le nickel.

Points de transformation. - Les points compris entre 600 et 675 sont extrêmement peu visibles; on voit un très léger pointement dans la courbe. Au contraire, les points compris entre 770 et 790 à l'échauffement, et 730 à 750 au refroidissement, sont d'une netteté remarquable; ils sont caractérisés par un très beau palier.

La dernière transformation, voisine de gooe, est plutôt une transformation lente.

B. Aciers trempés, - Micrographie. Les aciers trempés présentent la même structure que les aciers ordinaires à même teneur en carbone, les conditions du traitemement restant constantes.

Propriétés mécaniques. - Le tableau suivant résume les propriétés mécaniques des aciers trempés à $87^{\circ}$ dans l'eau à $+20^{\circ}$.

$\begin{array}{cc}\text { Composition } & \mathrm{Ta} \\ 0,120 & 0,09 \\ 0,170 & 0,15 \\ 0,180 & 0,60 \\ 0,160 & 1,05\end{array}$

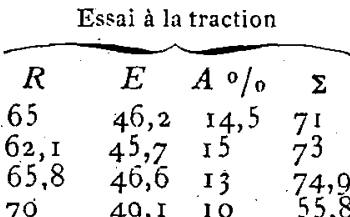

Essai
au choc Essai
a la bille

L'influence de la présence de très faibles quantités de tantale se fait sentir un peu plus dans les aciers trempés que dans les aciers recuits. 